Article

\title{
Structural and Optical Characteristics of PVA:C-Dot Composites: Tuning the Absorption of Ultra Violet (UV) region
}

\author{
Shujahadeen B. Aziz ${ }^{1,2^{*}}$, Aso Q. Hassan ${ }^{3}$, Sewara J. Mohammed ${ }^{4}$ \\ 1 Prof. Hameeds Advanced Polymeric Materials Research Lab., Department of Physics, College of Science, \\ University of Sulaimani, Qlyasan Street, Sulaimani, Kurdistan Regional Government/Iraq \\ 2 Komar Research Center (KRC), Komar University of Science and Technology, Sulaimani, 46001, Kurdistan \\ Regional Government/Iraq \\ 3,4 Department of Chemistry, College of Science, University of Sulaimani, Qlyasan Street, Sulaimani, \\ Kurdistan Regional Government/Iraq
}

\begin{abstract}
In this work the influence of carbon nano-dots (CNDs) on absorption of ultra violet (UV) spectra in hybrid PVA based composites was studied. The FTIR results reveal the complex formation between PVA and CNDs. The shifting was observed in XRD spectrum of PVA:CNDs composites compared to pure PVA. The strong absorption was observed in the absorption spectra of the composite samples. The intense absorption peaks appeared at $280 \mathrm{~nm}$ and $330 \mathrm{~nm}$ are ascribed to $\mathrm{n}-\pi^{*}$ and $\pi-\pi^{*}$ transition. The absorption edge shifted to lower photon energy sides with increasing CNDs. The refractive index increased linearly with increasing CNDS concentration. The optical dielectric constant which is related to the density of states was studied. The optical dielectric loss parameter was used to study the optical band gap of the samples. Taucs model was used to determine the type of electronic transition in the samples.
\end{abstract}

Keywords: carbon nanodots; hybrid polymer composites; FTIR study; XRX study; optical properties

\section{Introduction}

Since the invention of carbon nano-tubes (CNTs), carbon-based nano-materials have been widely investigated. Carbon quantum dots (CQDs) represent at the moment the newest class of carbon-based materials as a potential alternative to CNTs for sustainable applications [1]. Pyrolytic approaches have been proved to be an easy production method with high yield of CQDs. The past decade has seen a great research interest in fluorescent carbon nano-particles due to their outstanding optical properties, water dispersibility, and biocompatibility [2]. Quasi-spherical carbon nanoparticles with sizes below $10 \mathrm{~nm}$, termed carbon dots (CDs), are a fascinating class of luminescent nano-materials discovered in 2004 during the purification of single-walled carbon nano-tubes. They have attracted tremendous attention in various fields [3]. The abundance of oxygen/hydrogencontaining species such as $-\mathrm{OH}$ and $-\mathrm{COOH}$ on the surfaces of CQDs, they are opted as fillers to enhance the hydrogen bonding [4]. Lately, carbon nanodots (CNDs) have emerged as a new family of light-harvesting materials with remarkable advantages including strong and broad optical absorption, high chemical stability, excellent electron- and hole-transfer capability, and low toxicity [5]. The incorporation of CDs within polymer matrices are under intense study and thus can be utilized in many photonic and optoelectronic applications and integrated in real devices [6].Organicinorganic composites are designed for new eras of optical, nonlinear optical, electronic devices and biological labels [7]. The unique physicochemical properties and broad applications of polymer nano- 
composites have made them attracted to research. Furthermore, the presence of quantum dots or nano-particles in the solid polymer matrix will prevent the aggregation of nano-particles and thus store easier compared to the colloidal counterparts [8].Prior to apply organic composites into the devices their chemical structure, as well as morphological and optical properties have to be investigated. This is because any changes in the structure and properties of these materials can modify their performances [9]. Poly(vinyl alcohol) (PVA) is an interesting synthetic polymer with being non-toxic and water-soluble. This polymer has a high dielectric constant and an excellent filmforming ability. In addition, PVA possesses numerous functional groups on its backbone that make hydrogen bonding and thus facilitate the formation of polymer composites. The availability of a large number of those reactive functional groups and the hydrophilic nature make PVA easier for crosslinking with doping materials [10,11]. Owing to high-potential applications in light emitting devices, highly transparent and luminescent CDs/polymer composites have been a focus of research-anddevelopment in the past decade. Specifically, CD semiconductors are superior among the others in terms of light stability and low toxicity [12]. However, luminescence-quenching process induced by the particle aggregation limits the application of CDs concerning color tunability and white light fabrication in solid-state illumination systems. To prevent the aggregation of $\mathrm{CD}$ particles, they can successfully be combined with polar polymers [13]. In the present work, the composites are fabricated using PVA as hosting polymer since it is polar, thermo-stable, chemical resistant, easy processable and transparent [8].Conductive polymers have been the subject of research interest in recent years to understand their nature of charge transport whilst their optical properties are still rare. In order to fabricate polymer composites, different ratios of CDs are dispersed within PVA host polymer.

\section{Synthesis of CDs and preparation of polymer composites}

PVA used in this study was supplied by Sigma-Aldrich. PVA:CDs polymer nanocomposite films were prepared by the well-known solution casting technique. Hydrothermal treatment of lactose resulted in the formation of the yellow carbon nanodots (CNDs), as follows: $1 \mathrm{~g}$ of lactose was dissolved in $5 \mathrm{ml}$ of concentrated phosphoric acid and the resulting solution was colorless. The solution was then heated in a water bath at $(80-90){ }^{\circ} \mathrm{C}$ for (20-30) minutes until a dark brown solution was obtained. After cooling down to room temperature and adjusting the $\mathrm{pH}$ between (3-4) with dilute $\mathrm{NaOH}$, the solution was left overnight. Chloroform was used for the purification of CNDs. For the preparation of PVA solution, $1 \mathrm{~g}$ of PVA powder was dissolved in $100 \mathrm{ml}$ of distilled water. The solution was left under stirring at room temperature for 24 hours until the polymer was completely dissolved. The resulting solution became clear and viscous. To prepare the polymer nano-composite films, different amounts of CD were added individually into the PVA solution under continuous stirring. The polymer nano-composite samples were coded as CND0, CND1 and CND2 for PVA incorporated with $0 \mathrm{ml}, 15 \mathrm{ml}$, and $30 \mathrm{ml}$ of $5 \mathrm{mg}$ of dissolved CDs, respectively. The mixtures were stirred further until homogeneous solutions were achieved. After casting in different Petri dishes, the solutions were left for drying at room temperature to allow the films to be formed. The film thickness in the range of $120-121 \mu \mathrm{m}$ was controlled by casting the same amount of PVA. The films were transferred into desiccators for further drying. This procedure creates solvent-free films.

\section{Characterization techniques}

X-raydiffraction (XRD) data were collected at room temperature using a diffractometer( Bruker AXS GmbH) operating at a voltage of $40 \mathrm{kV}$ and a current of $40 \mathrm{~mA}$. The samples were scanned with a monochromatic $X$-radiation beam of wavelength $\lambda=1.5406 \mathrm{~A}^{\circ}$ and the glancing angles were in the range of $5^{\circ} \leq 2 \theta \leq 80^{\circ}$ with a step size of $0.1^{\circ}$. UV-vis absorption spectra were measured on a Jasco V-570 UV-Vis-NIR spectrophotometer r(Jasco SLM-468, Tokyo, Japan) in the absorbance mode. The formation of CNDs-PVA complexes was investigated by Fourier-transform infrared (FTIR) spectroscopy. FTIR spectra were recorded on a Nicolet iS10 FTIR spectrophotometer (Thermo Fischer Scientific, Waltham, MA, USA) in the wavenumber range of $4000-400 \mathrm{~cm}^{-1}$ with a resolution of $2 \mathrm{~cm}^{-1}$.

\section{Results and Discussion}




\subsection{FTIR Study}

FTIR analyses were used to investigate the complex formation in the samples. The FTIR spectra of pure PVA and all prepared PVA:CND composites are depicted in figure 1. As can be seen, the peak centered at $3322 \mathrm{~cm}^{-1}$ corresponding to the stretching vibration of hydroxyl groups $(\mathrm{O}-\mathrm{H})$ of pure PVA [14] was shifted to 3296 and $3312 \mathrm{~cm}^{-1}$, respectively, in CND1 and CND2 doped samples. Peak broadening and decreasing intensity can also be observed, indicating the complex formation of PVA and CNDs particles. Furthermore, the $\mathrm{CH}$ stretching of $\mathrm{CH}_{2}$ at an absorption of $2936 \mathrm{~cm}^{-1}$ in pure PVA [15], was shifted to 2930 and $2931 \mathrm{~cm}^{-1}$ in CND1 and CND2, respectively. The CH bending peakof pure PVA appearing at $1412 \mathrm{~cm}^{-1}$ [15], was shifted to 1408 and $1411 \mathrm{~cm}^{-1}$, respectively, in CND1 and CND2 composites. T he peak occurring at $1087 \mathrm{~cm}^{-1}$ in PVA assigned to C-O bending and stretching vibrations of acetyl group present on the polymer backbone [16, 17], was shifted to 1078 and $1079 \mathrm{~cm}^{-1}$ in the respective samples. The vibration peaks observing at between $842 \mathrm{~cm}^{-1}$ and 841 $\mathrm{cm}^{-1}$ are attributed to $\mathrm{C}-\mathrm{H}$ rocking mode or $\mathrm{C}-\mathrm{C}$ stretching $[16,18]$. The weak absorption bands can be seen at 1740 and $1370 \mathrm{~cm}^{-1}$, which represent $\mathrm{C}=\mathrm{O}$ stretching vibration and stretching vibration of methyl group in acetate moiety $\left(-\mathrm{COO}-\mathrm{CH}_{3}\right)$, demonstrating an incomplete alcoholysis [19]. A sharp peak seeing at $1561 \mathrm{~cm}^{-1}$ can be ascribed to skeletal $\mathrm{N}-\mathrm{H}$ bending mode [20]. These changes in the spectral features confirm a good cross-linking between PVA and CND nano-particles.
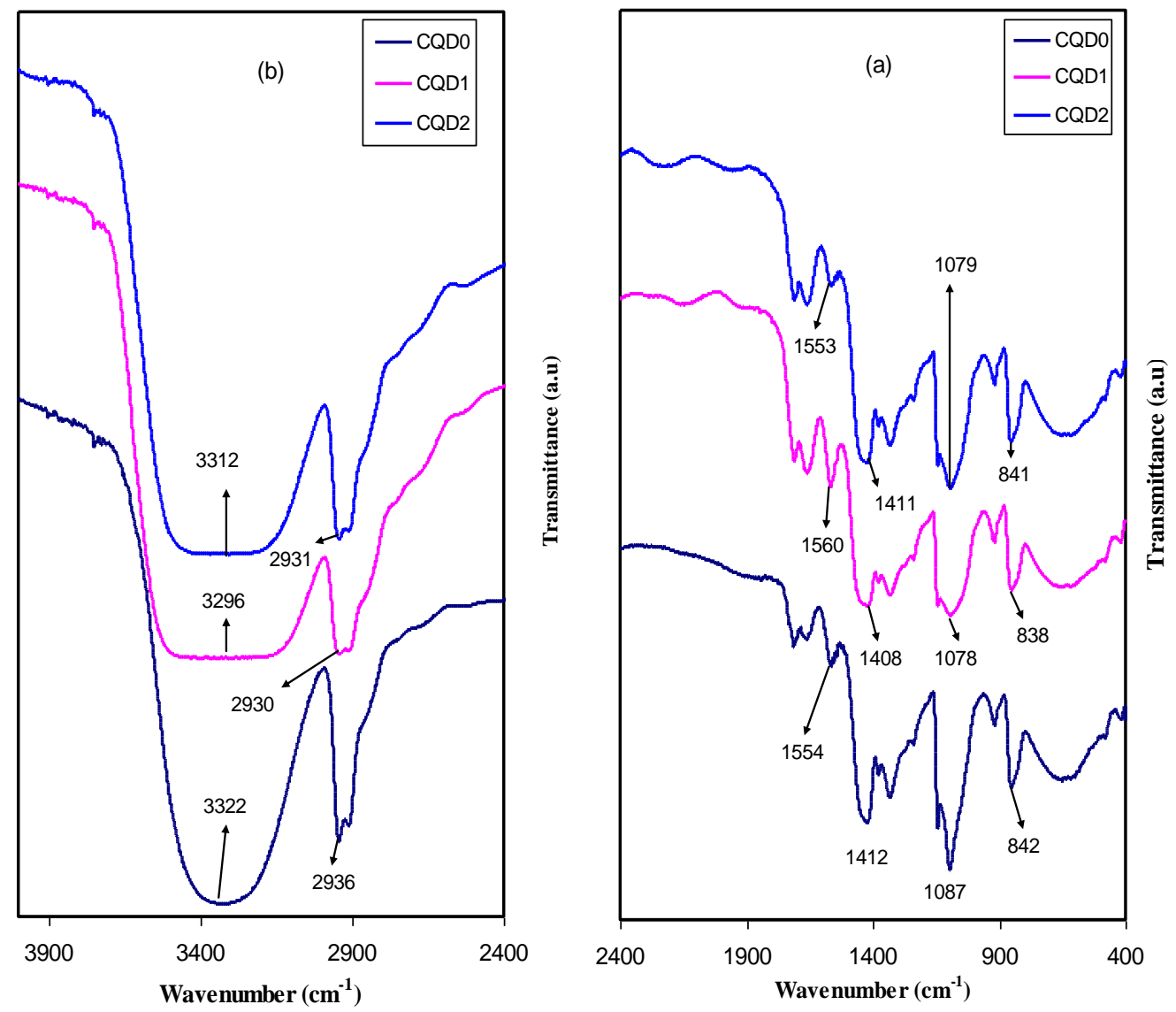

Figure 1. FTIR spectra of all samples in the region of (a) $400 \mathrm{~cm}^{-1}$ to $2400 \mathrm{~cm}^{-1}$, and (b) $2400 \mathrm{~cm}^{-1}$ to $4000 \mathrm{~cm}^{-1}$. Clear shifting, broadening and change in intensity in the FTIR bands can be observed.

\subsection{XRD Study}

Figure 2 shows the XRD spectrum of CND particles. It is clear that CDs exhibits a broad crystalline peak at about $2 \theta=27.97^{\circ}$ and a broad amorphous peak at $42.63^{\circ}$. Previous studies attributed the former peak to highly disordered carbon atoms [21]. Figure 3 represents the XRD pattern of pure PVA and PVA doped with CNDs particles. As can be seen, a broad peak at around 
$2 \theta=20^{\circ}$ in pure PVA corresponds to the semi-crystalline nature of the polymer [22]. In addition to this, two broad peaks at $2 \theta=23.4^{\circ}$ and $41.18^{\circ}$ are identified. In the literature, the typical diffraction peaks at $2 \theta=20^{\circ}, 23.43^{\circ}$ and $41.15^{\circ}$ are assigned to (101), (200) and (111) crystalline planes of PVA [19] and their shifts in the composite samplesare thus an evidence of complex formation between the functional groups of PVA and surface groups of CNDs particles. In this study, when the concentration of CNDs was increased, the intensity of the diffraction peak of pure PVA was decreased opposed to the peak broadness. This can be resulted from the disturbance of the polymer crystallinity through the disrupted hydrogen bonding between surface groups of CNDs particles and hydroxyl groups in PVA polymer which thus dominates an amorphous phase [14, 23]. No peaks for CNDs particles are existed in PVA doped samples demonstrating an entire dissolution of CNDs in polymer matrix.

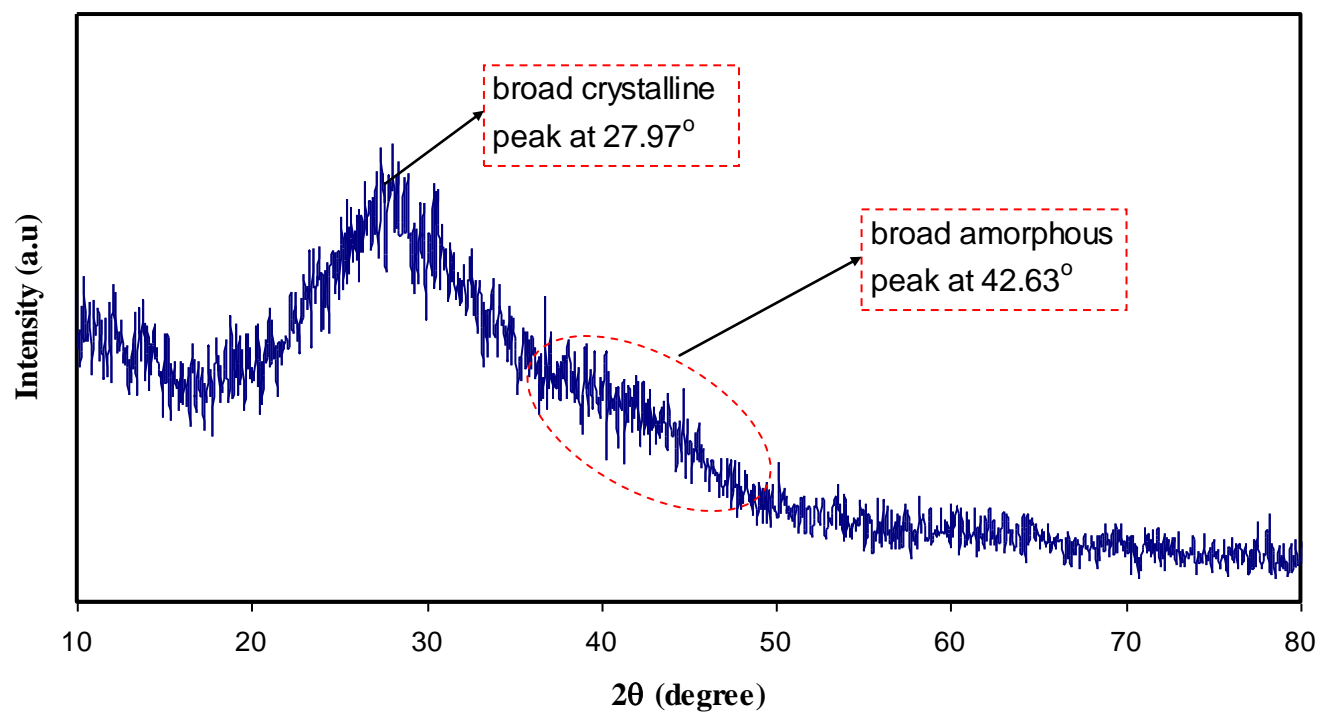

Figure 2. XRD pattern of $\mathrm{CN}$-dots at ambient temperature. Crystalline and amorphous peaks can be seen in the XRD spectrum.

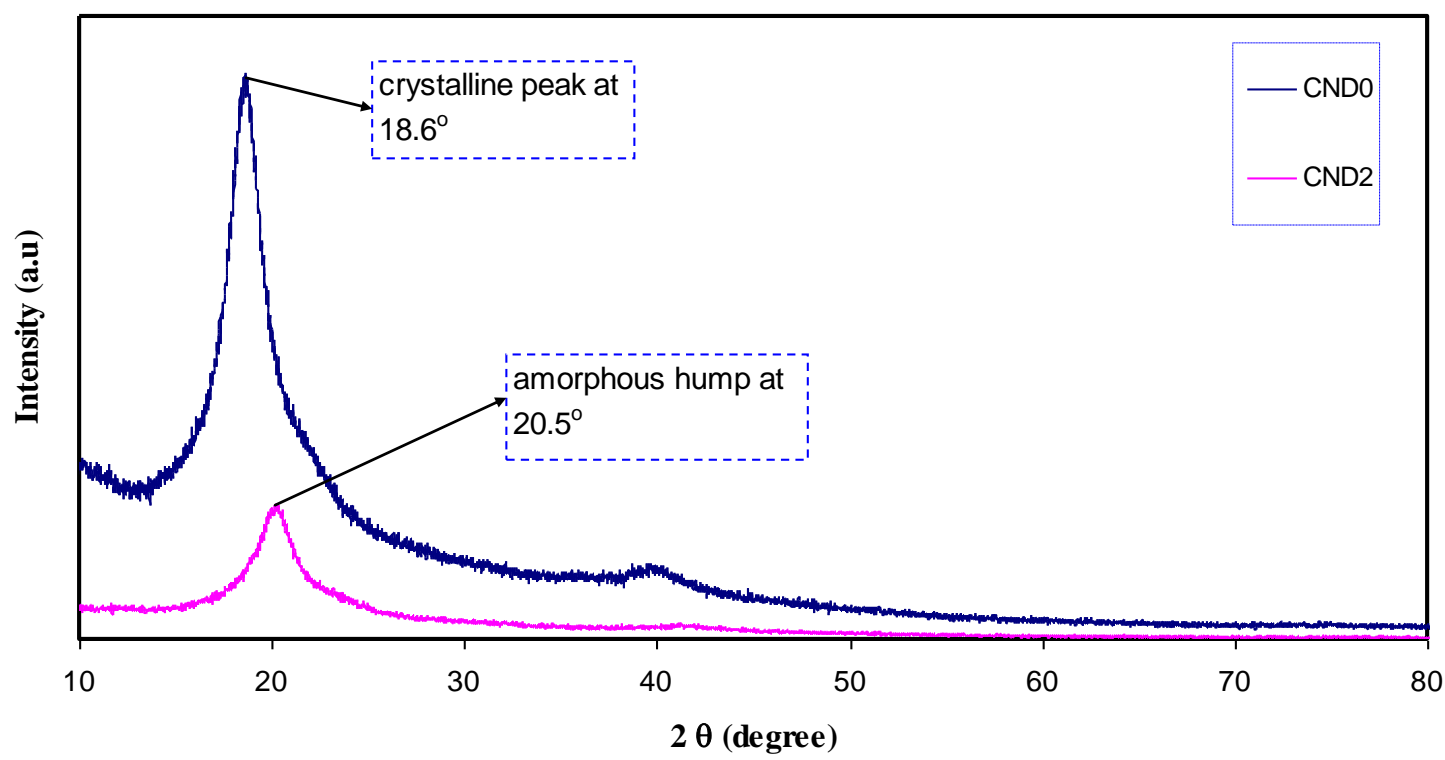

Figure 3. XRD pattern of pure PVA and PVA:CN-Dot composite films. It is interesting to note that the main peak of PVA is more broadened and it intensity decreased after incorporation of $\mathrm{CN}$-dots. 


\subsection{Absorption study}

In figure 4, the absorption spectra of pure PVA and PVA:CND films are illustrated. Two distinguished peaks can be manifested in the UV region. In comparison with pure PVA, the absorption of UV region is clearly tuned for the doped samples. The absorption of light energy by polymer materials in the UV and visible regions leading to promote electrons in $\sigma, \pi$ and norbitalsfrom the ground state to higher energy states is described by molecular orbital. The following electronic transitions are possible: $\sigma \rightarrow \sigma^{*}, \mathrm{n} \rightarrow \pi^{*}$, and $\pi \rightarrow \pi^{*}$. The presence of impurities causing most of the optical transitions has energies in the visible part of the spectrum. Therefore, color centers as defects are generated [24]. From Figure 4, two peaks at 280 and $330 \mathrm{~nm}$ (i.e. a broad shoulder peak) are detected in the spectrum which are due to the $n-\pi^{*}$ and $\pi-\pi^{*}$ transitions, respectively [25-27]. The unique optical and electronic properties of CDs particles allow applications in various fields such as biosensors, imaging probes, viral capsids, QD-based lasers, light emitting devices (LEDs) and photovoltaic cells [28]. It is apparent that onset of absorption for PVA:CND composites is from 580 $\mathrm{nm}$ lying in the visible region. CDs with their tunable absorption in the lower-mid region of the visible spectrum are important for applications in the range of optoelectronics and sensors to new formulations of bioimaging assays [29]. It is interesting to note that the absorption intensities of $n-\pi^{*}$ and $\pi-\pi^{*}$ transitions are increased by increasing CND concentrations. This can be related to the abundance of hydroxyl and amino functional groups on CND surface $[4,9,27]$.

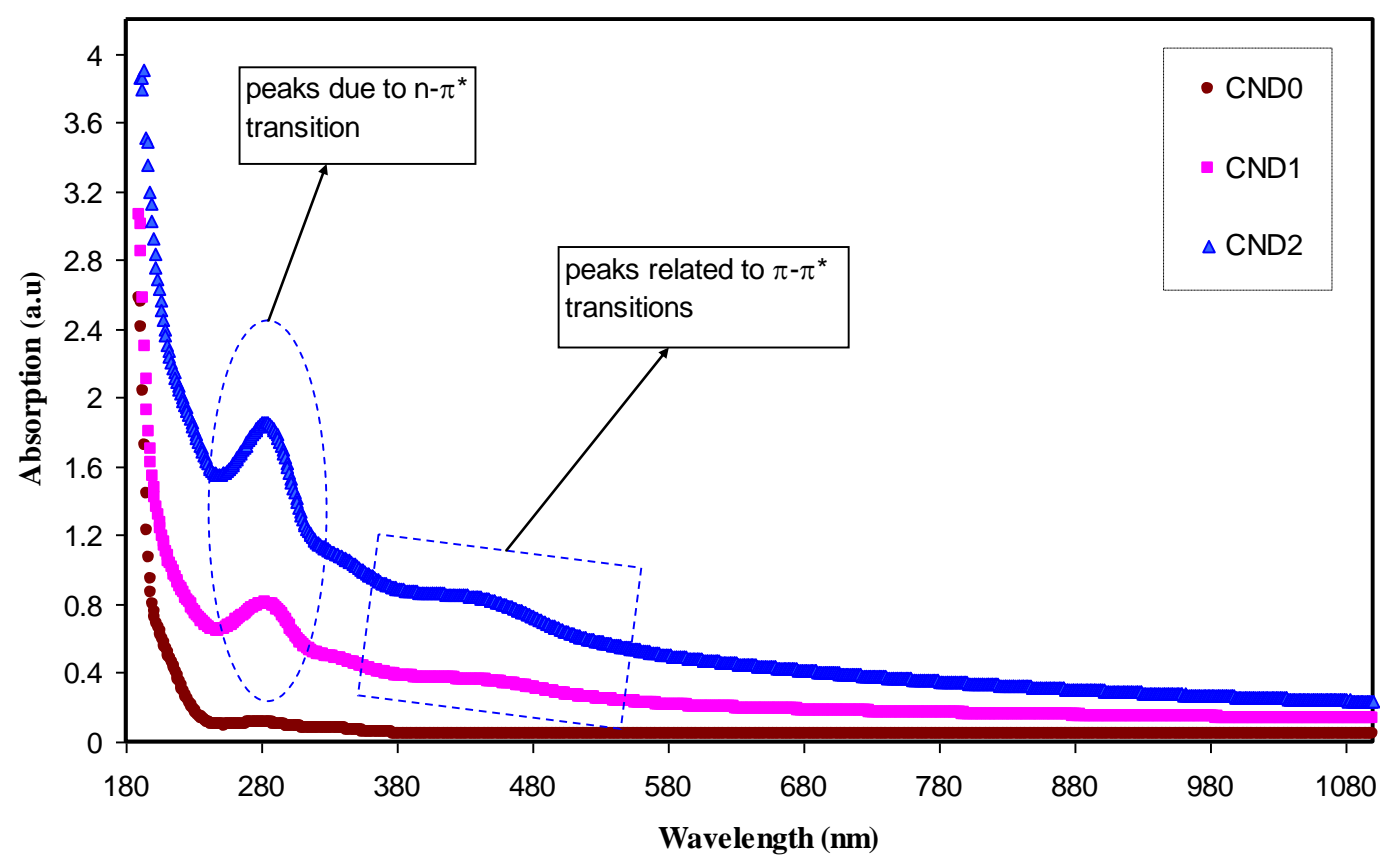

Figure 4. Absorption spectra for all the films. Clearly with increasing CNDs concentration the absorption shifts to higher wavelengths.

UV-vis is an informative technique for studying the electronic transitions. Band strength or band-gap energy can be measured from absorption edge in non-crystalline materials [30]. The absorption edge is a region in which an electron is jumped from a lower energy state to a higher energy state by an incident photon. The following equation was used to calculate the optical absorption coefficient from the transmittance and reflectance spectra of the films [31]:

$$
\alpha=\frac{1}{t} \operatorname{Ln}\left(\frac{T}{(1-R)^{2}}\right)
$$

where $t, T$ and $R$ are the thickness, transmittance and reflectance of the sample, respectively. Gradually increasing absorption coefficient with applied photon energies indicates the amorphous 
nature of the samples [31]. Figure 5 presents the absorption coefficient as a function of photon energy for pure PVA and PVA:CND films. A clear red shift from $6.2 \mathrm{eV}$ to $5.3 \mathrm{eV}$ corresponds to the absorption edge. An increase in CND concentration the absorption edge shifts towards lower photon energy. The shift in absorption edge might be resulted from the formation of conjugated bond system caused by bond cleavage and reconstruction. This supports the structural and chemical modifications of PVA incorporated with CQDs [32].

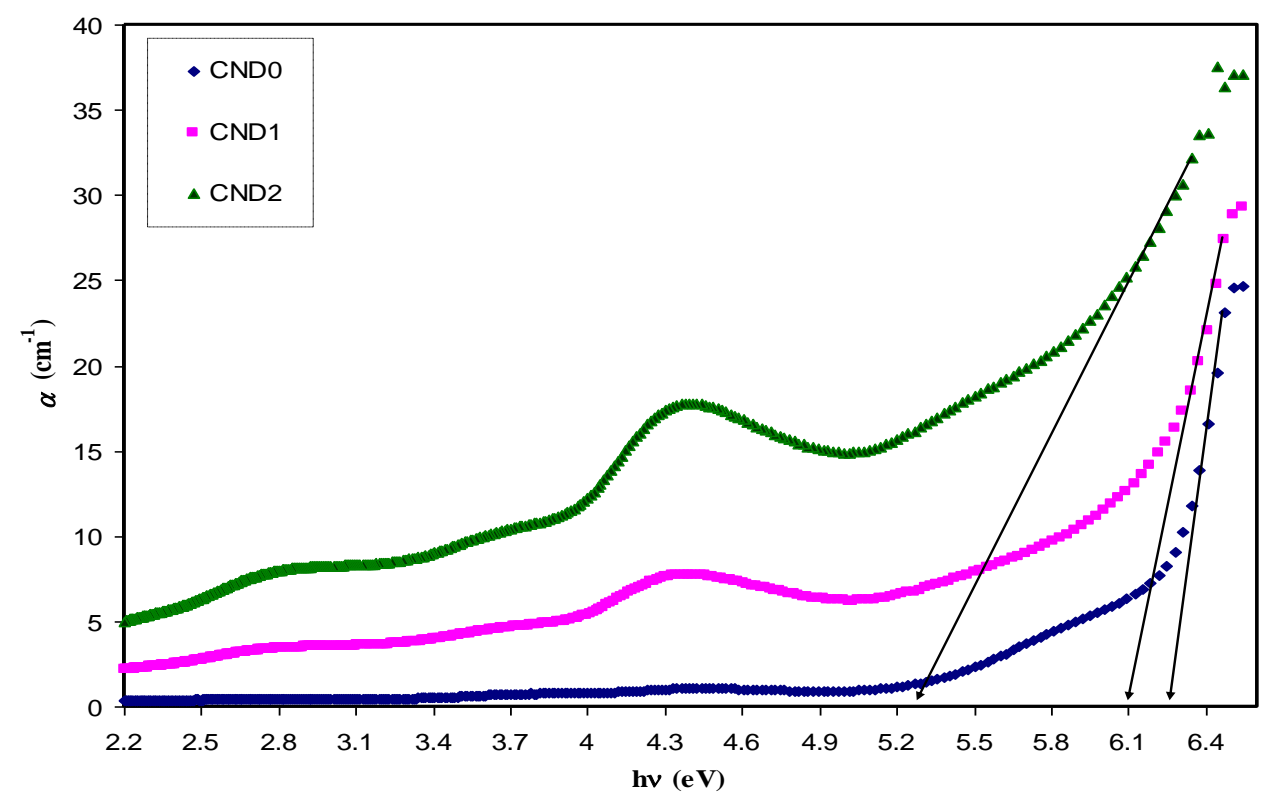

Figure 5. Absorption coefficient versus photon energy for all the films. Clearly with increasing CNDs concentration the absorption edge shifts lower photon energy.

\subsection{Refractive index and optical dielectric constant studies}

Today, studies on the electrical and optical properties of polymers have a great deal in view of their applications in optical devices with remarkable reflection, antireflection, interference and polarization properties. The optical properties of polymers can be properly modified by the addition of dopants depending on their reactivity with the host matrix [33-35]. One of the parameters is the optical refractive index (n), which is the measure of the reduction rate of the speed of light in the medium. The refractive index of the samples has been calculated from the reflectance (R) and extinction coefficient (k) by using the following equation [34],

$$
n=\left[\frac{(1+R)}{(1-R)}\right]+\sqrt{\frac{4 \times R}{(1-R)^{2}}}-K^{2}
$$

The refractive index spectra of pure PVA and the doped samples are illustrated in figure 6. It is obvious that an increase in the value of refractive index is related to an increase in CND concentration. The refractive index is greater than one since photons travelling through a material are slowed down by the interaction with electrons. The more photons are slowed down through the PVA:CND composites, the greater the refractive index's value will be. In principle, any factor causes to increase electron density in a material, also increase the refractive index [36]. Furthermore, the refractive index dispersion behavior as a function of wavelength can be observed for all the doped films compared to pure PVA as a result of increasing density. Two typical peaks seen in the refractive index spectra of the composite samples corresponds to aromatic $C=C \pi-\pi^{*}$ transition and $C=O n-\pi^{*}$ transition, respectively [37]. In addition, by increasing the CND concentration the peak intensity increases due to the abundance of more electron density and number of surface groups. In order to estimate the refractive index of the films, long wavelength region was extended to Y-axis. In figure 7, the refractive index is plotted against CND concentration. The linear dependence revealed in various studies 
indicates a good dispersion of fillers throughout the polymer matrix [18, 38- 41]. In the regression analysis, the value was found to be 0.99 from the best fitting data points. This confirms a homogeneous dispersion of CNDs throughout the PVA polymer. Refractive index is related to optical dielectric constant $(\varepsilon 1)$ parameter. This is directly related to the localized electronic states within the forbidden gap of materials $[18,22,42]$ :

$$
\varepsilon_{1}=n^{2}-k^{2}=\varepsilon_{\infty}-\frac{e^{2}}{4 \pi C^{2} \varepsilon_{o}} \frac{N}{m^{*}} \lambda^{2}
$$

where $\varepsilon_{\infty}$ and $\varepsilon_{o}$ refer to the dielectric constant at higher wavelengths and the free space dielectric constant, respectively. $\mathrm{N} / \mathrm{m}^{*}$ is the ratio of density of localized electronic states to the effective mass, $e$ the electronic charge, $\tau$ the optical relaxation time, $k$ the extinction coefficient and $C$ the velocity of light. Variation of the optical dielectric constant $\left(\varepsilon^{\prime}\right)$ with wavelength for different CND concentrations are represented in Figure 8. The $\varepsilon^{\prime}$ values are clearly increased with increasing CND concentration. An increase in $\varepsilon^{\prime}$ value from 1.3 to 2.4 is attributed to the increment of the density of states because of direct association of $\varepsilon^{\prime}$ parameter with the density of states inside the forbidden gap of the solid polymer films [22]. Relation between the static dielectric constant $\varepsilon^{\prime}(0)$ within long wavelengths and the band gap has been reported [43]. According to Penn model the optical dielectric constant can strongly be correlated with optical band gap $\left(E_{o}\right)[44]$ as follows:

$$
\varepsilon_{(o)} \approx 1+\left(\hbar \omega_{p} / E_{o}\right)^{2}
$$

However, this model can actually be connected to the refractive index (n) considering $\varepsilon=n^{2}$. Thus, the Penn model should be expressed in terms of refractive index [45].

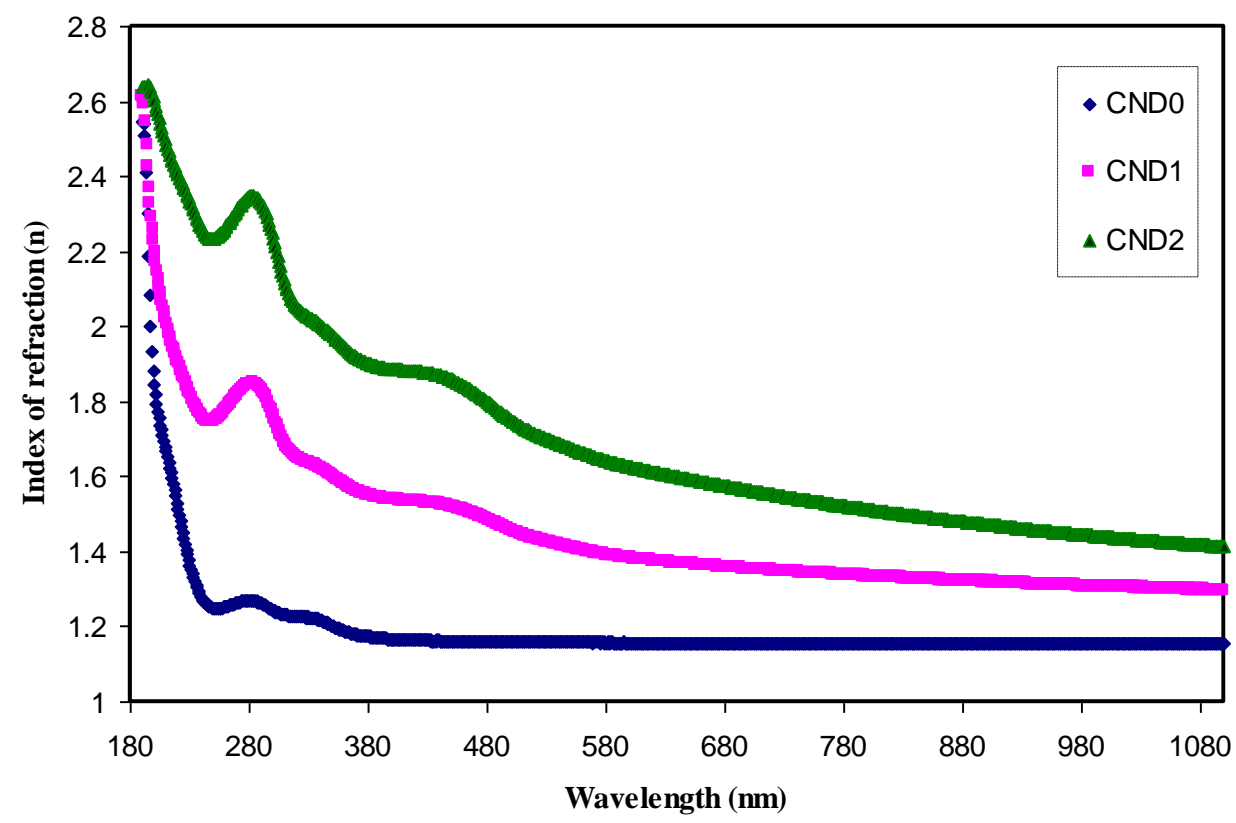

Figure 6. the index of refraction versus wavelength for all the films. Clearly with increasing CNDs concentration the dispersion increased. 


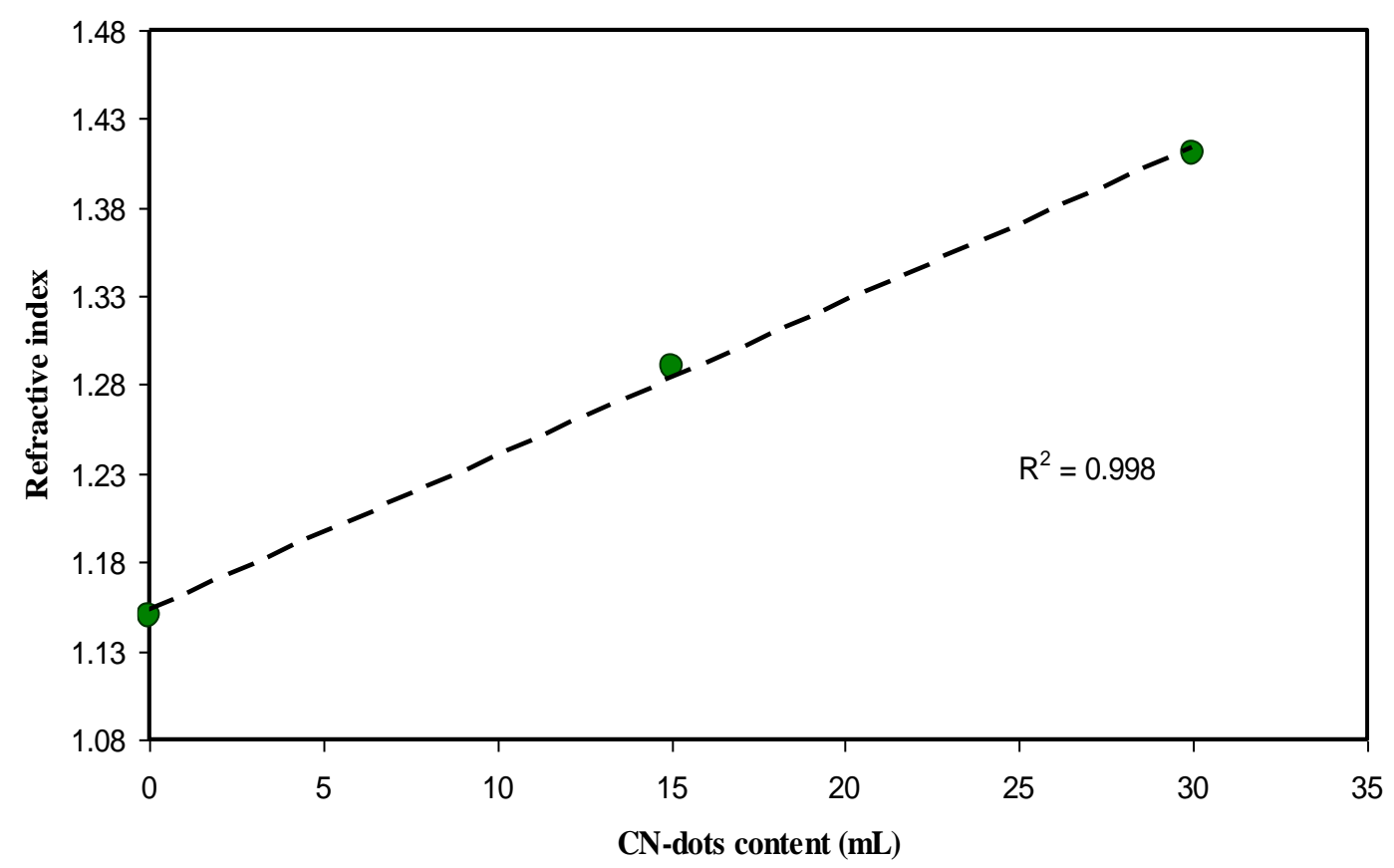

Figure 7. The index of refraction versus CNDs concentration. The linear increase reveals the homogeneous dispersion of CNDs particles.

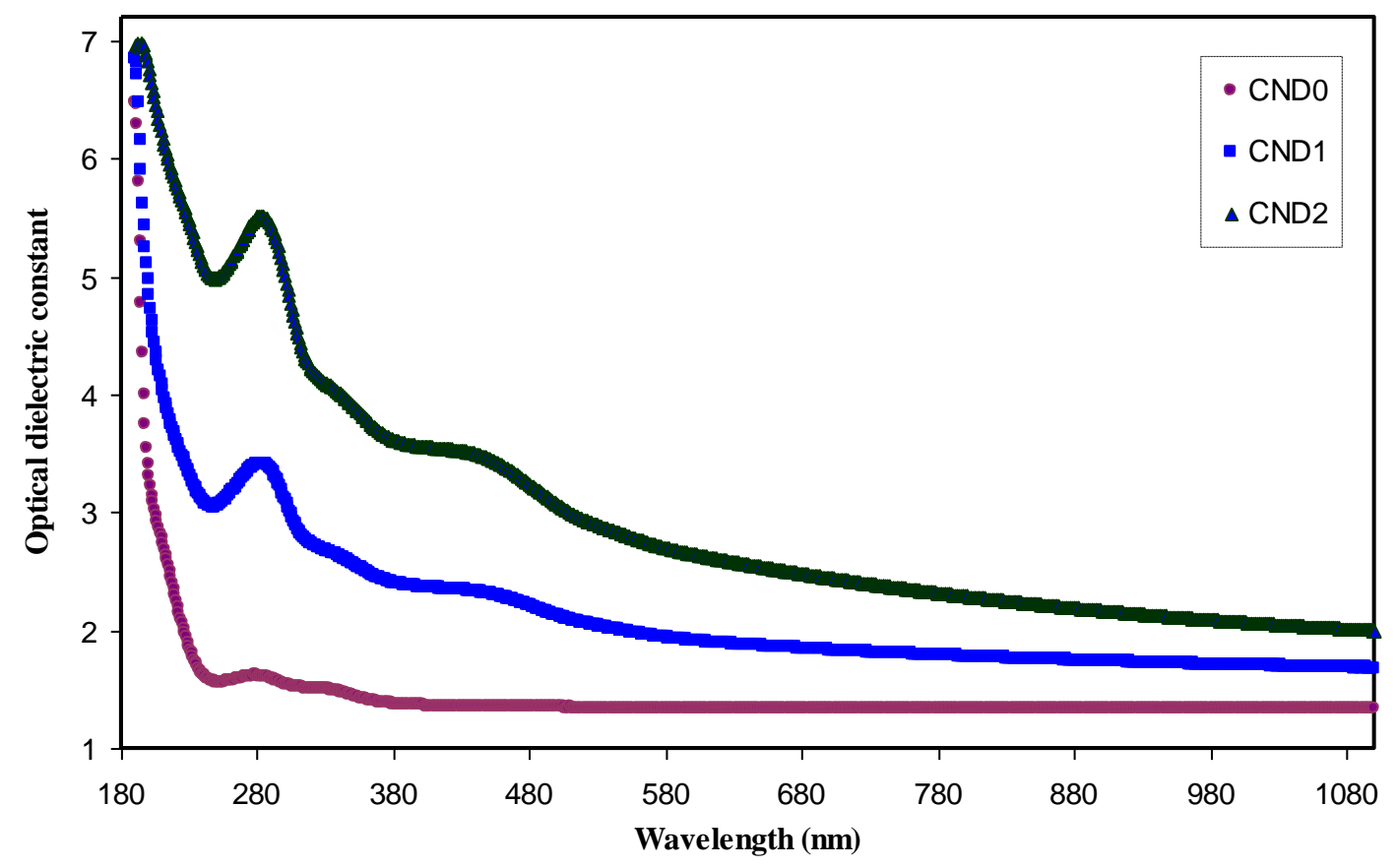

Figure 8. The optical dielectric constnat versus wavelength for all the films. Clearly with increasing CNDs concentration the $\varepsilon^{\prime}$ increased.

\subsection{Bandgap Study}

Interband absorption process deals with transition of electrons between the bands of solid materials. The absorption edge is originated from the onset of optical transitions across the fundamental band gap [46]. Our recent achievements revealed that the fundamental absorption edge derived from the dielectric loss represents the energy bandgap [18, 31, 33, 34, 40, 41, 47]. These are supported by quantum methods for bandgap investigations. Knowledge of both real and imaginary 
parts of the dielectric function allows calculating important optical functions [48]. The following complex optical dielectric function is usually used to describe the optical properties of a solid material, interrelated with photon and electron interactions [49].

$$
\varepsilon^{*}=\varepsilon 1(\omega)+j \varepsilon 2(\omega)
$$

$\varepsilon_{11}(\omega)$ as real part and $\varepsilon_{2}(\omega)$ as imaginary part of the complex are associated with electronic polarizability and electronic absorption of the material, respectively [50]. To better understand the electronic structure of different materials, the optical functions have to be studied. Frequencydependent dielectric function is closely linked to the electronic band structure. Accordingly, the optical properties of homogeneous mediums at all photon energies can be characterized [51]. From the quantum mechanical aspect, the transitions between occupied and unoccupied states are highly related to optical dielectric loss parameter $[18,31,33,34,40,41,47,51]$.The equation below can directly be used to determine the imaginary part $\varepsilon_{2}(\omega)$ of the complex dielectric function from the momentum matrix elements between the occupied and the unoccupied electronic states.

$$
\varepsilon_{2}=\frac{2 \pi e^{2}}{\Omega \varepsilon_{o}} \sum_{v, c, k}\left|\left\langle\Psi_{k}^{c}|\vec{u} \cdot \vec{r}| \Psi_{k}^{v}\right\rangle\right|^{2} \delta\left(E_{k}^{c}-E_{k}^{v}-\hbar \omega\right)
$$

where $\omega$ is the frequency of light, e the electronic charge, $\vec{u}$ the vector defining the polarization of the incident electric field, and $\left(E_{k}^{c}\right)$ and $\left({ }^{v}\right)$ the conduction and valence band wave functions at $k$, respectively [51].The fundamental absorption edge provides the optical band gap [52]. A rapid rise near the absorption edge can be a direct evidence for band gap determination [53, 54]. The main contributions to the optical spectra are derived from the top valence band (VB) to the lower conduction bands (CB), known as the fundamental absorption edge. The critical points obtained are associated with the band-gap values [49]. Metallic, semiconducting or insulating characteristics of a material can be determined from the electronic properties [55]. In figure 9, the optical dielectric loss is plotted against photon energy for all the samples. The intersection of linear part of $\varepsilon_{2}$ with the photon energy axis was utilized toestimate the optical band gaps. The values estimated from the plot are listedin Table 1.Going to the higher CQD concentration the band gap is reduced. Furthermore, Tauc's method was applied to specify the type of electronic transition. Optical absorption spectrum is significant for studying the physical properties of polymers includingthe study of a band construction and electronic properties when at pure and doped states [56].In fact, the optical bandgapwas estimated from the data of absorption coefficient versus wavelength using Tauc's equation.

$$
\alpha h v=B(h v-E g) n
$$

where $\alpha, h v, B$ and $E_{g}$ denote the absorption coefficient, the photon energy, the band form parameter and the optical bandgap of the samples, respectively, and $n$ a constant, being related to the density of states distribution and determined the type of transition. The $n$ values are equal to $1 / 2$ and $3 / 2$ for direct allowed and forbidden transitions, respectively, while $n$ values of 2 and 3 are for the case of indirect allowed and forbidden transitions, respectively [47]. A direct transition proceeds when the electrons wave vector remains unchanged. However, the interaction with a lattice vibration occurs in the indirect transition, in which the lowest region of the $\mathrm{CB}$ locates atdifferent part of the kspace from the highest region of the VB [56]. Figures 10 to 12 illustrate the plot of $(\alpha h v)^{1 / n}$ versus photon energy $(h v)$ when $n=3 / 2,2$ and 3. From these figures, various optical band gaps can be estimated as summarized in Table 1. Variations in the band gap values make hard to identify a dominant type of electronic transition. In order to specify the exact type of electronic transition in the samples, the band gaps obtained from Tauc's method (i.e. figures 10 to 12) were compared to the optical band gaps derived from optical dielectric loss plot (i.e. figure 9). As a result of comparison, the type of forbidden direct transition (i.e. $\mathrm{n}=3 / 2$ ) can be deduced. A decrease in $E_{g}$ upon increasing CND concentration might result from the creation of higher number of free charge carriers/radicals [32]. To determine the band gap and understand the electron transition phenomena from the top of 
VB to the bottom of CB both optical dielectric loss parameter and Tauc's model have to be tested. In our previous works, the use of optical dielectric loss parameter for studying the bandgap has been well established [18, 31, 33, 34, 40, 41, 47, 56]. Thus, the present work is an additional support to our hypothesis implying that optical dielectric loss parameter and Tauc's model are sufficient to study the band gap and electron transition types, respectively.

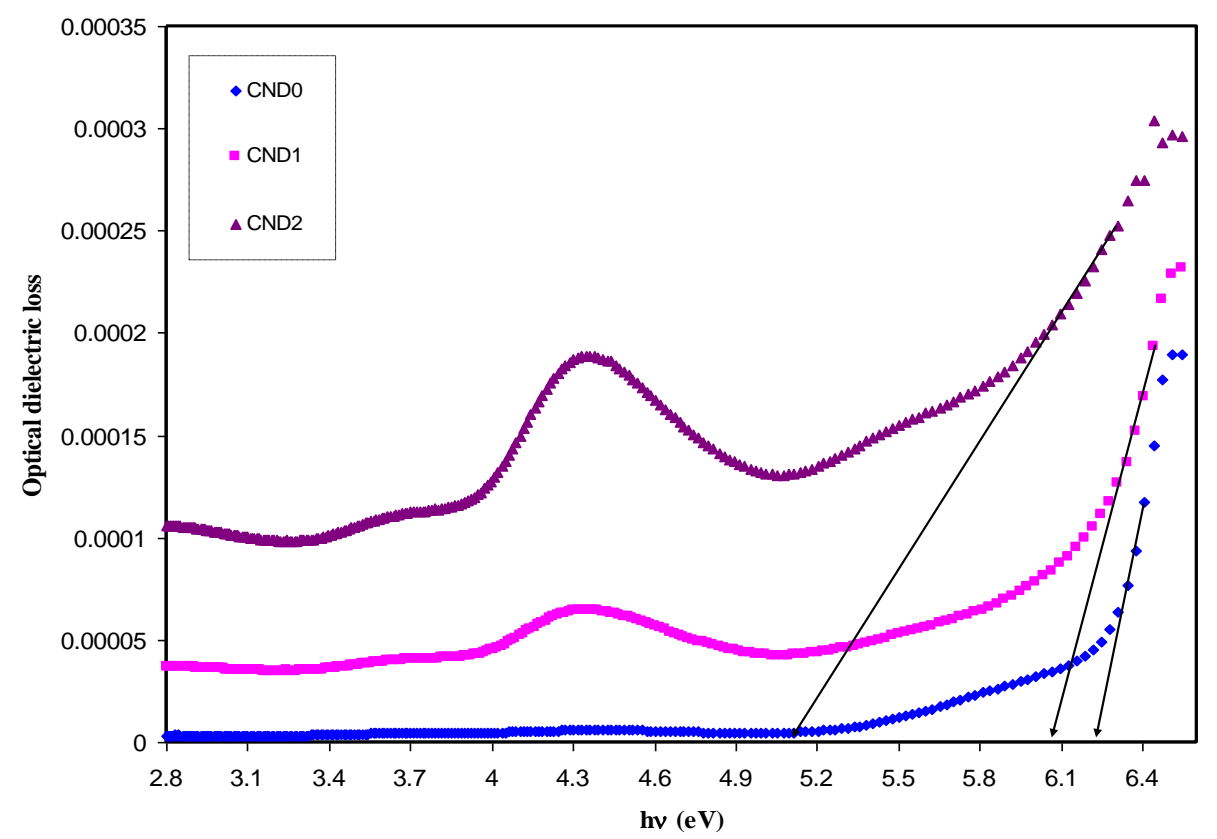

Figure 9. optical dielectric loss versus photon energy (hv) for all samples. Distinguishable linear parts can be manifested at high photon energy region which can be used to estimate the optical band gap.

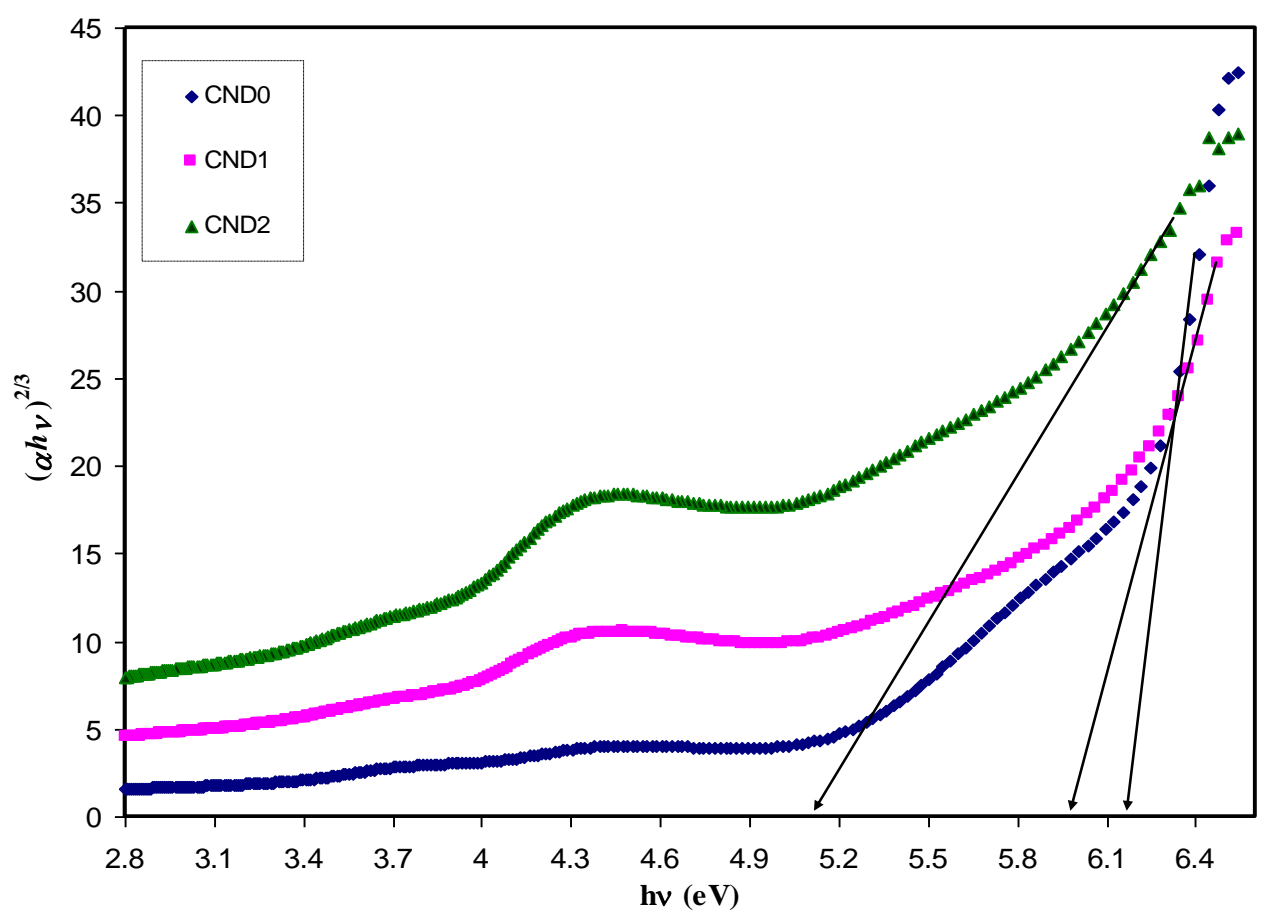

Figure 10. Plot of $(\boldsymbol{\alpha h v})^{2 / 3}$ versus photon energy $(\boldsymbol{h} \boldsymbol{v})$ for all the samples 


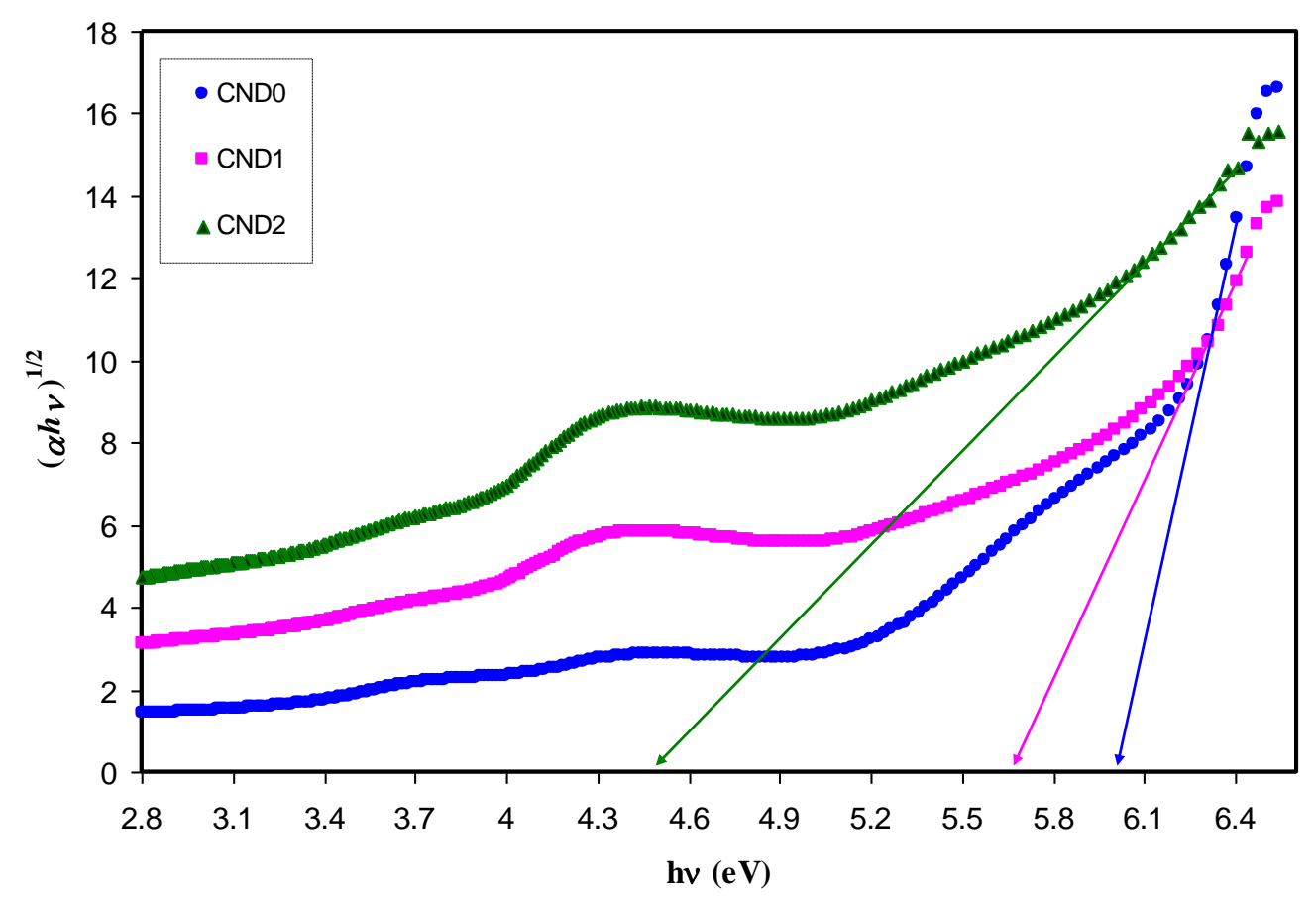

Figure 11. Plot of $(\boldsymbol{\alpha h v})^{1 / 2}$ versus photon energy (hv) for all the samples

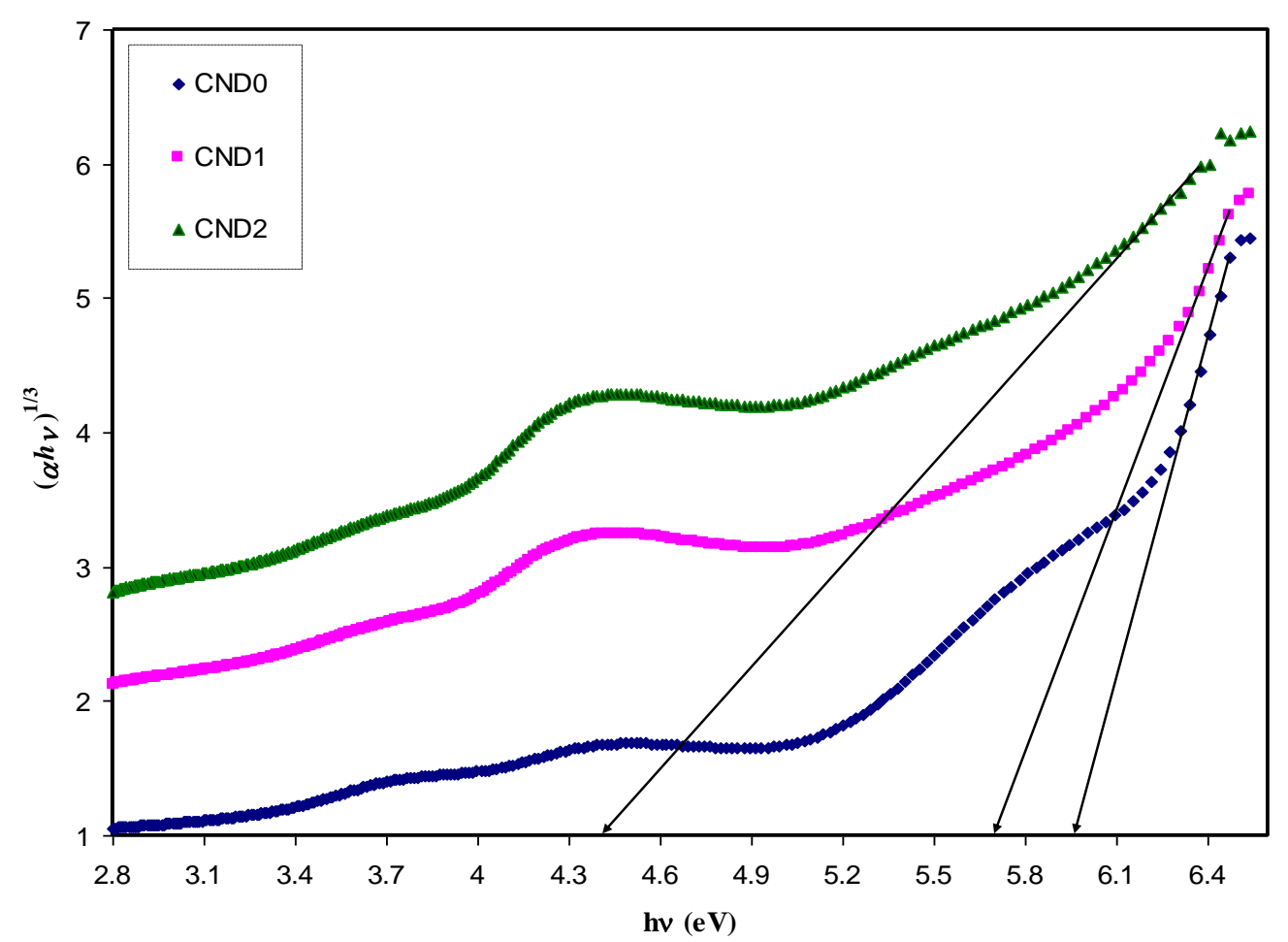

Figure 12. The plots of $(\alpha h v)^{\wedge / 3}$ vs $(h v)$ for all the samples. 
Table 1. estimated bandgap from Taucs model and optical dielectric loss plot.

\begin{tabular}{ccccc}
\hline $\begin{array}{c}\text { Sample } \\
\text { designation }\end{array}$ & $\begin{array}{c}\text { Eg }(\mathrm{eV}) \text { from Tauc } \\
\text { method }(\mathrm{n}=3)\end{array}$ & $\begin{array}{c}\text { Eg }(\mathrm{eV}) \text { from } \\
\text { Tauc method }(\mathrm{n} \\
=2)\end{array}$ & $\begin{array}{c}\text { Eg }(\mathrm{eV}) \text { from } \\
\text { Tauc method }(\mathrm{n} \\
=3 / 2)\end{array}$ & $\begin{array}{c}\text { Estimated bandgap } \\
\text { from } \varepsilon^{\prime \prime} \text {-hv plot }\end{array}$ \\
CND 0 & 5.95 & 6 & 6.18 & 6.2 \\
CND 1 & 5.7 & 5.67 & 5.96 & 6.05 \\
CND 2 & & 5.12 & 5.12 \\
\hline
\end{tabular}

\section{Conclusions}

In this research work, fabrication of PVA using CNDs has been investigated. The polymer composites were characterized by FTIR, XRD, and UV-vis spectroscopy. Changes in the FTIR spectral features indicated a good cross-linking between PVA and CND nano-particles. Shifts in XRD spectra of the composites confirmed the complex formation between them. Furthermore, the effect of these nano-particles on tuning the absorption of UV spectra in the nano-composites was studied. Strong absorptions at 280 and $330 \mathrm{~nm}$ were assigned to $n-\pi^{*}$ and $\pi-\pi^{*}$ transition. A reduction in the optical band gap was resulted from a shift in absorption edge to lower photon energy. A linear increase of refractive index with increasing CND concentration revealed a homogeneous distribution of the particles throughout the host PVA. Optical dielectric loss parameter was utilized to estimate the optical band gap. The study of Tauc's model established a forbidden direct type of electronic transition. The results of the present work is an additional support to our hypothesis implying that optical dielectric loss parameter and Tauc's model are sufficient to study the bandgap and electron transition types, respectively.

Acknowledgment: The authors gratefully acknowledge the financial support for this study from the Department of Physics, College of Science, University of Sulaimani, Sulaimani, and Komar Research Center (KRC), Komar University of Science and Technology, Sulaimani, 46001, Kurdistan Regional Government, Iraq. The authors appreciatively acknowledge the financial support from the Kurdistan National Research Council (KNRC)- Ministry of Higher Education and Scientific Research-KRG/Iraq for this research project.

Conflicts of Interest: The authors declare no conflicts of interest regarding the publication of this work.

\section{References}

[1] K. N. Ambasankar, L. Bhattacharjee, S. K. Jat, R. R. Bhattacharjee, K. Mohanta "Study of Electrical Charge Storage in Polymer-Carbon Quantum Dot Composite" Chemistry Select 2 (2017) 4241-4247

[2] X. Zhang, S. Wang, C. Zhu, M. Liu, Y. Ji, L. Feng, L. Tao, Y. Wei " Carbon-dots derived from nanodiamond: Photoluminescence tunable nanoparticles for cell imaging " Journal of Colloid and Interface Science 397 (2013) $39-44$

[3] M. Amjadi, Z. Abolghasemi-Fakhri, T. Hallaj " Carbon dots-silver nanoparticles fluorescence resonance energy transfer system as a novel turn-on fluorescent probe for selective determination of cysteine " Journal of Photochemistry and Photobiology A: Chemistry 309 (2015) 8-14

[4] G. Yang, X. Wan, Y. Liu, R. Li, Y. Su, X. Zeng, J. Tang "Luminescent poly(vinyl alcohol)/carbon quantum dots composites with tunable water-induced shape memory behavior in different $\mathrm{pH}$ and temperature environments"ACS Appl. Mater. Interfaces, DOI: 10.1021/acsami.6b11476

[5] Yuri Choi, Dasom Jeon, Yeongkyu Choi, Jungki Ryu, and Byeong-Su Kim"Self-Assembled Supramolecular Hybrid of Carbon Nanodots and Polyoxometalates for Visible-Light-Driven Water Oxidation"ACS Applied Materials \& Interfaces 10 (2018) 13434-13441

[6] A. Kovalchuk, K. Huang, C. Xiang, A. A. Martí, J. M. Tour " Luminescent Polymer Composite Films Containing Coal-Derived Graphene Quantum Dots " ACS Appl. Mater. Interfaces, 2015, 7 (47), pp 26063-26068 
[7] C. Woelfle, R. O. Claus "Transparent and flexible quantum dot-polymer composites using an ionic liquid as compatible polymerization medium" Nanotechnology 18 (2007) 025402 (9pp)

[8] B. Suo, X. Su, J. Wu, D. Chen, A. Wang, Z. Guo "Poly (vinyl alcohol) thin film filled with CdSe-ZnS quantum dots: Fabrication, characterization and optical properties" Materials Chemistry and Physics 119 (2010) 237-242

[9] M. I. Azmer, Z. Ahmad, K. Sulaiman, F. Touati, " Morphological and structural properties of VoPcPhO:P3HT composite thin films " Materials Letters 164 (2016) 605-608

[10] S. B. Aziz, Omed Gh. Abdullah, S. A .Hussein, H. M Ahmed "Effect of PVA Blending on Structural and Ion Transport Properties of CS:AgNt-Based Polymer Electrolyte Membrane" Polymers 9 (2017) 622

[11] S. B. Aziz, R.T. Abdulwahid, M. A. Rasheed, O. Gh. Abdullah, H. M. Ahmed "Polymer Blending as a Novel Approach for Tuning the SPR Peaks of Silver Nanoparticles" Polymers 9 (2017) 486

[12] L. Chen, C. Zhang, Z. Du, H. Li, L. Zhang, W. Zou "Fabrication of amido group functionalized carbon quantum dots and its transparent luminescent epoxy matrix composites" J. APPL. POLYM. SCI. 132 (2015) 42667, DOI: 10.1002/APP.42667

[13] S. K. Bhunia, S. Nandi, R. Shikler, R. Jelinek "Tuneable light-emitting carbon-dot/polymer flexible films prepared through one-pot synthesis" Nanoscale 8 (2016) 3400-3406

[14] N. Ahad, E. Saion, E. Gharibshahi "Structural, Thermal, and Electrical Properties of PVA-Sodium Salicylate Solid Composite Polymer Electrolyte" Journal of Nanomaterials, Volume 2012, Article ID 857569, 8 pages, doi:10.1155/2012/857569

[15] P. B. Bhargav, V. M. Mohan, A. K. Sharma, V. V. R. N. Rao "Structural, Electrical and Optical Characterization of Pure and Doped Poly (Vinyl Alcohol) (PVA) Polymer Electrolyte Films" International Journal of Polymeric Materials, 56:579-591, 2007

[16] H. Gao, K. Lian "Characterizations of proton conducting polymer electrolytes for electrochemical capacitors" Electrochimica Acta 56 (2010) 122-127

[17] K. P. Radha, S. Selvasekarapandian, S. Karthikeyan, M. Hema, C. Sanjeeviraja "Synthesis and impedance analysis of proton-conducting polymer electrolyte PVA:NH4F" Ionics, DOI 10.1007/s11581-013-0866-5

[18] S. B. Aziz, M. A. Rasheed, A. M. Hussein, H. M. Ahmed "Fabrication of polymer blend composites based on [PVA-VP] $(1-x):(A g 2 S) x(0.01 \leq x \leq 0.03)$ with small optical band gaps: Structural and optical properties" Materials Science in Semiconductor Processing 71 (2017) 197-203

[19] L. Jiang, T. Yang, L. Peng, Y. Dan "Acrylamide modified poly(vinyl alcohol): crystalline and enhanced water solubility"RSC Adv., 2015, 5, 86598-86605

[20] C.-W. Liew, K. H. Arifin, J. Kawamura, Y. Iwai, S. Ramesh, A.K. Arof "Electrical and structural studies of ionic liquid-based poly(vinyl alcohol)

proton conductors" Journal of Non-Crystalline Solids 425 (2015) 163-172

[21] K. M. Omer, A. Q. Hassan "Chelation-enhanced fluorescence of phosphorus doped carbon nanodots for multi-ion detection" Microchim Acta 184 (2017) 2063

[22] S. B. Aziz "Modifying poly (vinyl alcohol)(PVA) from insulator to small-bandgap polymer: A novel approach for organic solar cells and optoelectronic devices" Journal of Electronic Materials 45 (2016) 736-745

[23] S. B. Aziz, O. Gh. Abdullah, M. A. Rasheed "Structural and electrical characteristics of PVA: NaTf based solid polymer electrolytes: role of lattice energy of salts on electrical DC conductivity" J Mater Sci: Mater Electron 28 (2017) 12873

[24] R. Kumar, S. A. Ali, A. K. Mahur, H. S. Virk, F. Singh, S. A. Khan, D. K. Avasthi, R. Prasad "Study of optical band gap and carbonaceous clusters in swift heavy ion irradiated polymers with UV-Vis spectroscopy" Nuclear Instruments and Methods in Physics Research B 266 (2008) 1788-1792

[25] G. Yang, X. Wan, Y. Liu, R. Li, Y. Su, X. Zeng, J. Tang "Luminescent poly(vinyl alcohol)/carbon quantum dots composites with tunable water-induced shape memory behavior in different $\mathrm{pH}$ and temperature environments"

[26] X. Li, S. Zhang, S. A. Kulinich, Y. Liu, H. Zeng "Engineering surface states of carbon dots to achieve controllable luminescence for solid-luminescent composites and sensitive Be21 detection" SCIENTIFIC REPORTS | 4 : 4976 | DOI: 10.1038/srep04976

[27] Y. Wang, A. Hu "Carbon quantum dots: synthesis, properties and applications"J. Mater. Chem. C, 2 (2014) 6921-6939

[28] J. Weaver, R. Zakeri, S. Aouadib and P. Kohli " Synthesis and characterization of quantum dot-polymer composites " J. Mater. Chem., 2009, 19, 3198-3206 
[29] C. S. Stan, P. G. Horlescu, L. E. Ursu, M. Popa, C. Albu "Facile preparation of highly luminescent composites by polymer embedding of carbon dots derived from N-hydroxyphthalimide" Journal of Materials Science 52 (2017) 185-196

[30] S. A. Saq'an, A. S. Ayesh, A. M. Zihlif, E. Martuscelli, G. Ragosta "Physical properties of polystyrene/alum composites" Polymer Testing 23 (2004) 739-745

[31] S. B. Aziz, O. Gh. Abdullah, A. M. Hussein, H. M. Ahmed "From Insulating PMMA Polymer to Conjugated Double Bond Behavior: Green Chemistry as a Novel Approach to Fabricate Small Band Gap Polymers"Polymers 9 (2017) 626

[32] K. Hareesh, G. Sanjeev, A. K. Pandey, V. Rao "Characterization of UV-irradiated Lexan polycarbonate films" Iran Polym J (2013) 22:341-349

[33] S. B. Aziz, H. M. Ahmed, A. M. Hussein, A. B. Fathulla, R. M. Wsw, R. T. Hussein "Tuning the absorption of ultraviolet spectra and optical parameters of aluminum doped PVA based solid polymer composites" J Mater Sci: Mater Electron 26 (2015) 8022

[34] S. B. Aziz, R. T. Abdulwahid, H. A. Rsaul, H. M. Ahmed "In situ synthesis of CuS nanoparticle with a distinguishable SPR peak in NIR region" J Mater Sci: Mater Electron (2016) 27: 4163

[35] S. B. Aziz, O. Gh. Abdullah, A. M. Hussein, R. T. Abdulwahid, M. A. Rasheed, H. M. Ahmed, S. W. Abdalqadir, A. R. Mohammed "Optical properties of pure and doped PVA: PEO based solid polymer blend electrolytes: two methods for band gap study" J Mater Sci: Mater Electron (2017) 28: 7473

[36] K. E. Babu, A. Veeraiah, D. T. Swamy, V. Veeraiah "First-principles study of electronic and optical properties of cubic perovskite CsSrF3" Materials Science-Poland, DOI: 10.2478/s13536-012-0047-7

[37] Y. Zhou, S. K. Sharma, Z. Peng, R. M. Leblanc "Polymers in Carbon Dots: A Review" Polymers 9 (2017) 67; doi:10.3390/polym9020067

[38] J. Jin, R. Qi, Y. Su, M. Tong, J. Zhu, "Preparation of high-refractive-index PMMA/TiO2 nanocomposites by one-step in situ solvothermal method", Iran Polym. J. 22 (2013) 767-774

[39] P. Tao, Y. Li, A. Rungta, A. Viswanath, J. Gao, B. C. Benicewicz, R. W. Siegel, L. S. Schadler, "TiO2 nanocomposites with high refractive index and transparency", J. Mater. Chem. 21 (2011) 18623-18629

[40] S. B. Aziz, M. A. Rasheed, H. M. Ahmed "Synthesis of Polymer Nanocomposites Based on [Methyl Cellulose](1-x):(CuS) x (0.02 M $\leq x \leq 0.08 \mathrm{M})$ with Desired Optical Band Gaps"Polymers 9 (2017) 194

[41] S. B. Aziz "Morphological and Optical Characteristics of Chitosan (1-x):Cuox $(4 \leq \mathrm{x} \leq 12)$ Based Polymer Nano-Composites: Optical Dielectric Loss as an Alternative Method for Tauc's Model" Nanomaterials 7 (2017) 444

[42] I. Saini, J. Rozra, N. Chandak, S. Aggarwal, P. K. Sharma, A. Sharma, "Tailoring of electrical, optical and structural properties of PVA by addition of Ag nanoparticles", Mater. Chem. Phys.139 (2013) 802-810

[43] Z. E. Biskri, H. Rached, M. Bouchear, D. Rached, M. S. Aida, "A Comparative Study of Structural Stability and Mechanical and Optical Properties of Fluorapatite (Ca5(PO4)3F) and Lithium Disilicate (Li2Si2O5) Components Forming Dental Glass-Ceramics: First Principles Study", J. Electron. Mater. 45 (2016) 5082-5095

[44] N. M. Ravindra, P. Ganapathy, J. Choi, "Energy gap-refractive index relations in semiconductors - An overview", Infrared Phys. Technol. 50 (2007) 21-29

[45] M. F. Plass, C. Popov, B. Ivanov, S. Mänd, M. Jelinek, L. M. Zambov, W. Kulisch, "Correlation between photoluminescence, optical and structuralproperties of amorphous nitrogen-rich carbon nitride films", Appl. Phys. A 72 (2001) 21-27

[46] S. B. Aziz, M. A. Rasheed, Z. H. Z. Abidin " Optical and electrical characteristics of silver ion conducting nanocomposite solid polymer electrolytes based on chitosan" Journal of Electronic Material 46 (2017) 6119. https://doi.org/10.1007/s11664-017-5515-8

[47] S. B. Aziz, S. M. Mamand, S. R. Saed, R. M. Abdullah, S.A. Hussein, "New Method for the Development of Plasmonic Metal-Semiconductor Interface Layer: Polymer Composites with Reduced Energy Band Gap" J. Nanomater. 2017 (2017) Article ID 8140693 (2017)

[48] H. Cui-E, Z. Zhao-Yi, C. Yan,C. Xiang-Rong, C. Ling-Cang "First-principles calculations for electronic, optical and thermodynamic properties of ZnS" Chinese Physics B 17 (2008) 3867-3874

[49] S. Cheddadi, K. Boubendira, H. Meradji, S. Ghemid, F. E. H. Hassan, S. Lakel, R. Khenata "First-principle calculations of structural, electronic, optical, elastic and thermal properties of $\mathrm{MgXAs} 2(\mathrm{X}=\mathrm{Si}, \mathrm{Ge})$ compounds"Pramana - J. Phys. (2017) 89:89

[50] T. B. Nasr , H. Maghraoui-Meherzi, H. Ben Abdallah, R. Bennaceur "First principles calculations of electronic and optical properties of Ag2S"Solid State Sciences 26 (2013) 65-71 
[51] Md. A. Rahman, Md. A. Rahman, U. K. Chowdhury, M.T.H. Bhuiyan, Md. L. Ali, Md. A. R. Sarker "First principles investigation of structural, elastic,electronic and optical properties of $\mathrm{ABi} 2 \mathrm{O} 6(\mathrm{~A}=\mathrm{Mg}$, $\mathrm{Zn})$ with trirutile-type structure" Cogent Physics (2016), 3: 1257414

[52] Z. Xin-Yin, W. Yue-Hua, Z. Min, Z. Na, G. Sai, C. Qiong "First-Principles Calculations of the Structural, Electronic and Optical Properties of BaZrxTi1-xO3 ( $x=0$, 0.25, 0.5, 0.75)" CHIN. PHYS. LETT. Vol. 28, No. 6 (2011) 067101

[53] W. Y. Ching, Z.-Q. Gu, Y.-N. Xu "First-principles calculation of the electronic and optical properties of LiNb03" Phys. Rev. B 50, 1992(R)

[54] S. B. Aziz, O. Gh. Abdullah, M. A. Rasheed "A novel polymer composite with a small optical band gap: New approaches for photonics and optoelectronics" J. Appl. Polym. Sci. 2017, 134, 44847

[55] X. Li, H. Cui, R. Zhang "First-principles study of the electronic and optical properties of a new metallic MoAlB" Scientific Reports 6 (2016) 39790, DOI: 10.1038/srep39790

[56] H. M. Zidan, M. Abu-Elnader, "Structural and optical properties of pure PMMA and metal chloride-doped PMMA films" Physica B 355 (2005) 308-317

(C) 2018 by the authors. Submitted for possible open access publication under the terms and conditions of the Creative Commons Attribution (CC BY) license (http://creativecommons.org/licenses/by/4.0/). 